\title{
Avalanche dynamics in fluid imbibition near the depinning transition
}

\author{
Marc Pradas, ${ }^{1}$ Juan M. López, ${ }^{2}$ and A. Hernández-Machado ${ }^{1}$ \\ ${ }^{1}$ Departament d'Estructura i Constituents de la Matèria, \\ Universitat de Barcelona, Avinguda Diagonal 647, E-08028 Barcelona, Spain \\ ${ }^{2}$ Instituto de Física de Cantabria (IFCA), CSIC-UC, E-39005 Santander, Spain
}

(Dated: December 4, 2018)

\begin{abstract}
We study avalanche dynamics and local activity of forced-flow imbibition fronts in disordered media. We focus on the front dynamics as the mean velocity $\bar{v}$ of the interface is decreased and the pinning state is approached. Scaling arguments allow us to obtain the statistics of avalanche sizes and durations, which become power-law distributed due to the existence of a critical point at $\bar{v}=0$. Results are compared with phase-field numerical simulations.
\end{abstract}

PACS numbers: 05.40.-a, 47.56.+r, 64.60.Ht, 68.35.Ct

In many physical systems, the response to a slow external driving usually involves avalanches or bursts. Different examples are found in fracture cracks [1], granular material [2], earthquakes [3], or during imbibition of fluids in porous media [4], among others. A particularly interesting problem in this context is the dynamics of fronts during imbibition of fluids in porous media [4], with its many engineering applications in fluidics and oil-recovery technology.

Imbibition in disordered media occurs when a viscous fluid, which wets the medium preferentially, displaces a less viscous fluid (typically air) and therefore, at relatively low injection rates, stable fronts separating the two phases are formed (for a recent review on imbibition see Ref. [5]). In the case of forced-flow imbibition, the spatially averaged velocity of the liquid-air interface $\bar{v}$ is kept constant by means of a constant injection rate. Then, for relatively low velocities, the invading fluid advances in the form of spatially localized events or avalanches, as occurs in other disordered systems.

Avalanche dynamics in imbibition is expected to be responsible for the front velocity fluctuations. Rost et al. [4] have recently shown that in the case of imbibition, which is a locally conserved process, velocity fluctuations are controlled by a length scale $\xi_{\times}$arising from fluid conservation. This characteristic length scale introduces a natural cutoff in the distribution of avalanche sizes and durations, which leads to non-critical avalanche distributions and is ultimately responsible for the lack of correlated fluctuations at large distances. The scaling behavior of the velocity fluctuations can then be derived making use of the central limit theorem [4]. Forcedflow imbibition described by avalanches with a fixed cutoff size has been experimentally observed in the recent work by Planet et al. [6], by means of analyzing the global velocity time series $\bar{v}(t)$.

In this paper we study the statistics of local avalanches of activity in forced-flow imbibition in disordered media. We analyze the mesoscopic behavior of the interface by monitoring locally active sites, i.e. those sites that are moving at a given time, which define the actual avalanche taking place in the system (see Fig. 11). We show that avalanche sizes and durations become power-law distributed for low enough injection rates due to the existence of a critical point at $\bar{v}=0$.


FIG. 1: (Color online) (a) Typical avalanches of the front $h(x, t)$ from our phase-field simulations for $\bar{v}=v_{0} / 40$ and clip $c_{\mathrm{th}}=3$. (b) Spatio-temporal activity is characterized by the size $s$, lateral extent $\ell$, and duration $T$ of avalanches.

The singularity appearing as $\bar{v} \rightarrow 0$ affects the value of the critical exponents that characterize the front dynamics and morphology-namely, the avalanche exponents, as well as the roughness exponents. This leads to effective exponents even for finite velocities. We obtain scaling relations connecting the roughness exponents and the avalanche exponents. Our scaling theory is compared with numerical results of a phasefield model for imbibition.

Only in the last few years it has been possible to achieve a satisfactory theoretical understanding of imbibition, based on a detailed description of the physical forces that play a relevant role at different spatial scales [5]. Surface tension $\sigma$ tends to flatten the front at short length scales, while quenched disorder in both, capillary $p_{c}(\boldsymbol{r})$ and permeability $K(\boldsymbol{r})$, makes the front to roughen and fluctuate around its average position. Both disorders operate at very different length scales, separated by a crossover length that depends on the velocity as $\xi_{K} \sim 1 / \bar{v}[5,7]$. We are concerned here with the interesting case of a slowly advancing front, i.e. the capillary dominated regime, where the permeability disorder is irrelevant and can be considered to be a constant $K(\boldsymbol{r}) \sim K_{0}$. Different theoretical approaches [7-9] have arrived at the conclusion that, for small deviations around the mean interface position, the dynamical evolution for the liquid-air interface is described in Fourier space as

$$
\partial_{t} \hat{h}_{k}=-\sigma K_{0}|k| k^{2} \hat{h}_{k}-\bar{v}|k| \hat{h}_{k}+K_{0}|k| \hat{\eta}_{k},
$$

where $\hat{\eta}_{k}(\hat{\mathbf{h}})$ are the Fourier components of the capillary dis- 
order at some coarse-grained scale. From Eq. (1) one can easily see that there exists a crossover length

$$
\xi_{\times} \sim\left(\frac{\sigma K_{0}}{\bar{v}}\right)^{1 / 2}
$$

such that interface fluctuations are uncorrelated above this typical scale. Indeed, several numerical studies [8, 10, 12] have shown that the interface is asymptotically flat on length scales larger than $\xi_{\times}$, introducing then a natural cutoff in the system. For capillary-induced fluctuations we have $\xi_{\times} \ll \xi_{K}$, so that the permeability disorder can be ignored.

Avalanche statistics. - In order to monitor local avalanches of forward movements we proceed as follows. First, we define the active sites on the interface as those where the local velocity $v(x, t) \equiv \partial_{t} h(x, t)$ takes values above some fixed threshold, $v(x, t)>c_{\mathrm{th}} \bar{v}$, where $c_{\mathrm{th}}$ is some arbitrary constant and $\bar{v}$ is the spatially averaged global velocity. An avalanche is defined as a connected cluster of active $\left[v(x, t)>c_{\mathrm{th}} \bar{v}\right]$ sites surrounded by non-active $\left[v(x, t)<c_{\mathrm{th}} \bar{v}\right]$ sites (see Fig. 1).

Avalanches exhibit a typical size (volume) $\langle s(\ell)\rangle$ for an event of lateral spatial extent $\ell$. For a given front velocity $\bar{v}$ we expect the average avalanche size to scale with the lateral extent up to the cutoff length scale, $\langle s(\ell)\rangle \sim \ell^{D}$ for $\ell \ll \xi_{\times} \sim \bar{v}^{-1 / 2}$, where $D$ is the avalanche dimension exponent that can be easily related with the local roughness exponent $\alpha_{\text {loc }}$ via the local width of the interface fluctuations $w(\ell)$. One has $\langle s(\ell)\rangle \sim l^{d} w(\ell) \sim \ell^{d} \ell^{\alpha_{\text {loc }}}$, and $D=d+\alpha_{\text {loc }}$ in $d+1$ dimensions. In particular, for $d=1$, one observes that $\alpha_{\mathrm{loc}}=1[8-10,13]$ and then $D=2$. We also expect to observe a scaling relation $\langle s(T)\rangle \sim T^{\gamma}$ between an avalanche of duration $T$ and its size below a certain time cutoff $T_{\times}$.

In order to study the statistics of the avalanche dynamics, we first calculate the probability densities $\mathcal{P}(s)$ and $\mathcal{P}(T)$ for having avalanches of size $s$ and duration $T$, respectively. Due to the existence of the intrinsic crossover length in the imbibition problem the several avalanche probability distributions are not generically expected to be critical, but exponentially decaying functions:

$$
\mathcal{P}(\varrho) \sim \varrho^{-\tau_{\varrho}} \exp -\left(\varrho / \varrho_{\times}\right),
$$

where $\mathcal{P}(\varrho)$ is the marginal probability density function (PDF); the index $\varrho$ denotes the size $s$, lateral extent $\ell$, or duration $T$ of avalanches, and $\tau_{\varrho}$ is an exponent. The distribution cutoff depends explicitly on $\bar{v}$ and, in particular, the maximum avalanche size is given by

$$
s_{\times} \sim \xi_{\times}^{D} \sim(\bar{v})^{-D / 2} .
$$

This cutoff diverges $\left(\xi_{\times} \rightarrow \infty\right)$ as the control parameter $\bar{v} \rightarrow$ 0 , which renders critical avalanches expanding over the whole system. This divergence is very strong, $s_{\times} \sim(\bar{v})^{-1}$, already in $d=1$, which -in turn- is expected to be reflected in longtailed avalanche distributions even for finite values of $\bar{v}$ (see Fig. 2).

Let us now consider the joint probability $\mathcal{P}(s, \ell, T)$ for having an avalanche of size $s$, extent $\ell$, and duration $T$.
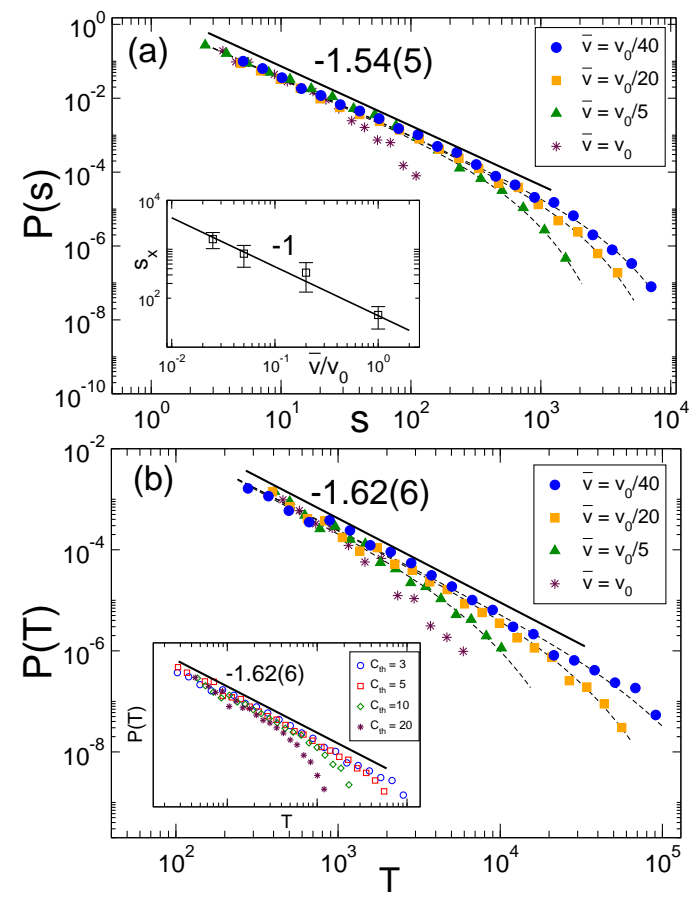

FIG. 2: (Color online) (a) Distribution of avalanche size for different velocities. The dashed curves correspond to a data fit to $\mathcal{P}(s)=a s^{-\tau_{s}} \exp \left[-s / s_{\times}(\bar{v})\right]$. The solid line fits the power-law regime for the smallest velocity. The inset shows the cutoff $s \times$ of the avalanche size distribution for each velocity with a guide-to-eye line of slope -1 . (b) Distribution of avalanche durations. The inset shows a comparison for different choices of the arbitrary threshold $c_{\mathrm{th}}$ from 3 to 20 in the case of $\bar{v}=v_{0} / 40=5 \times 10^{-5}$ a. u.

At the critical point $\bar{v}=0$ scale-invariant behavior implies $\mathcal{P}(s, \ell, T)=b^{\sigma} \mathcal{P}\left(b^{D} s, b \ell, b^{z} T\right)$ for any scaling factor $b>0$. Here, $z$ corresponds to the interface dynamic exponent. Integrating over two of the arguments one obtains the marginal PDFs and the scaling relations

$$
\tau_{T}=1+\frac{D}{z}\left(\tau_{s}-1\right), \quad \text { and } \quad \tau_{\ell}=1+z\left(\tau_{T}-1\right),
$$

that must be satisfied in the case of scale-invariant avalanche dynamics. They connect the avalanche activity exponents $\left(\tau_{T}, \tau_{s}, \tau_{\ell}\right)$ with the dynamics of the front $(z)$. Note that in this limit long-range interface correlations fully coincide with avalanches of correlated events. This basically means that an avalanche occupies a significant fraction of lateral extent of the system and cooperative correlated motion over large scales does occur. Accordingly, close to the pinning critical point we have $\langle\ell\rangle \sim T^{1 / z}$. These scaling relations immediately imply $\langle s\rangle \sim T^{D / z}$, so the exponent $\gamma$ relating avalanche sizes versus durations becomes

$$
\gamma=D / z=\left(d+\alpha_{\mathrm{loc}}\right) / z,
$$

in the limit $\bar{v} \rightarrow 0$.

This result is to be contrasted with the scaling relation obtained in a recent work by Rost et al. [4]. They analyzed a regime of relatively high velocities for which the 
length scale $\xi_{\times}$is very small as compared with system size $L$. In this regime avalanches are very narrow and one can decompose the front motion in independent, spatially localized, avalanches of forward moves, $\bar{v}_{\text {ava }} \sim\left(\bar{h} / \xi_{\times}\right)^{d} \bar{v}$. The avalanche duration is $T \sim w(\ell) / v(\ell)$, where $v(\ell)$ is the front velocity over the region of size $\ell$ spanned by the avalanche. If simultaneous avalanches are narrow and independent events a central limit theorem argument gives $v(\ell) \sim \ell^{-d / 2}$ and this leads to $\langle s(T)\rangle \sim T^{\gamma}$, with [4]

$$
\gamma=\left(\alpha_{\mathrm{loc}}+d\right) /\left(\alpha_{\mathrm{loc}}+d / 2\right),
$$

in $d+1$ dimensions. In particular, for $d=1$ one has the prediction $\gamma=4 / 3$ [4]. Interestingly, this argument also leads to the scaling relation $\langle\ell(T)\rangle \sim T^{\delta}$, with an exponent $\delta=1 /\left(\alpha_{\text {loc }}+d / 2\right)$ that differs from $1 / z$, where $z=3$ is the dynamic exponent describing the correlation spreading of interfacial fluctuations for forced-flow imbibition in $d=1[9,10,12,13]$. This indicates that the propagation of interface correlations is decoupled from the avalanche dynamics. Indeed, as it will be shown below, the scaling theory leading to Eq. (7) is valid in a velocity regime such that the characteristic length scale is negligible as compared with the system size $\xi_{\times} \ll L$. For lower front velocities, when $\xi_{\times}$becomes comparable with the system size, the exponent $\gamma$ should tend to the value given by Eq. (6) instead of (7).

Scaling properties in the static limit.- The interface scaling exponents can be obtained by a scaling theory which is expected to be valid in the static (pinned state) limit $\bar{v} \rightarrow 0$. In the pinned state the velocity-dependent term in Eq. (1) cancels and the geometric properties of the front can be described by the balance between surface tension and capillary disorder, which in real space can be written as $\sigma \nabla^{2} h_{\mathrm{p}}(x)+\eta(x) \simeq 0$, where $h_{\mathrm{p}}(x)$ is the pinned state, and the disorder is delta correlated, $\left\langle\eta(x) \eta\left(x^{\prime}\right)\right\rangle=\eta_{0}^{2}+\Delta^{2} \delta\left(x-x^{\prime}\right)$, with a mean value $\langle\eta(x)\rangle \equiv \eta_{0}$ and variance $\Delta^{2}-\eta_{0}^{2}$. Applying a scaling transformation, $x \rightarrow b x$ and $h_{\mathrm{p}} \rightarrow b^{\alpha} h_{\mathrm{p}}$, scale-invariance holds for a global roughness exponent $\alpha=3 / 2$ for the pinned state configuration. Small perturbations of the pinned state $\delta h$ are assumed to relax towards another of the infinitely many pinned configurations according to

$$
\partial_{t}(\delta h)=\sigma K_{0} \nabla^{2}(\delta h)+K_{0} \eta(x),
$$

which leads to the exact interface exponents $z=2, \alpha=3 / 2$, and $\alpha_{\mathrm{loc}}=1$ at the critical point $\bar{v}=0$ for $d=1$. These exponents can now be replaced in the avalanche scaling relations (5) and (6) to obtain $\tau_{T}=\tau_{s}, \tau_{\ell}=2 \tau_{T}-1$, and $\gamma=1$, where we have used $D=d+\alpha_{\mathrm{loc}}=2$ in $d=1$. In the following we compare these scaling results with numerical integrations of a phase-field model as one approaches the singular point $\bar{v}=0$.

The phase-field model.- The scaling properties of fluid imbibition fronts can be well described by means of a phasefield model [8, 9]. A conserved field $\phi$ is used to represent the two existing phases, taking the equilibrium values $\phi_{e q}=+1$ and $\phi_{e q}=-1$ in the liquid and air phases, respectively.

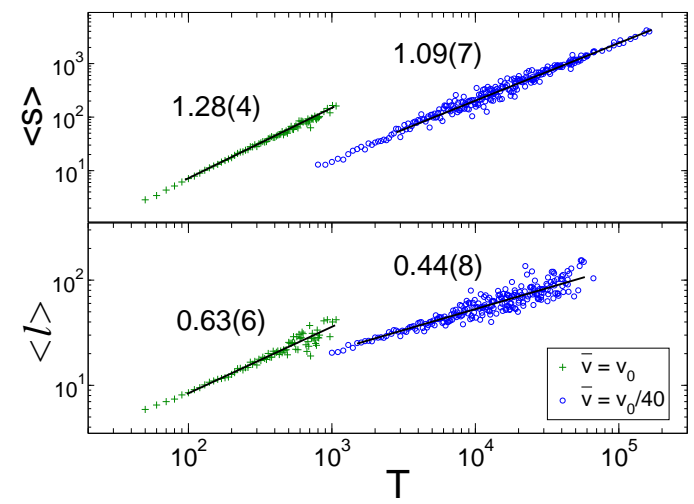

FIG. 3: (Color online) Average avalanche size and lateral extent versus duration in a system of size $L=512$ for two velocities in the different dynamical regimes discussed in this paper.

The dynamics of the phase field is controlled by a continuity equation based on a time-dependent Ginzburg-Landau model with conserved order parameter $\partial_{t} \phi=\nabla M \nabla \mu$ where $\mu=$ $\delta \mathcal{F} / \delta \phi$ is the chemical potential and the free energy takes the form $\left.\mathcal{F}[\phi]=\int d \boldsymbol{r}\left[(\epsilon \boldsymbol{\nabla} \phi)^{2} / 2\right)-\phi^{2} / 2+\phi^{4} / 4-\eta(\boldsymbol{r}) \phi\right]$. The quenched random field $\eta(\boldsymbol{r})>0$ models capillary disorder and favors the liquid (wet) phase, forcing the interface to advance at the expense of the air (dry) phase. In our numerical model we have used a spatially distributed dichotomic quenched noise in a two-dimensional system. The locally conserved dynamics is described by

$$
\partial_{t} \phi=\nabla M \nabla\left[-\phi+\phi^{3}-\epsilon^{2} \nabla^{2} \phi-\eta(\boldsymbol{r})\right],
$$

where $M$ is a mobility parameter which we take constant at the liquid phase $(\phi>0)$ and zero at the air phase $(\phi<0)$, and the disorder is Gaussian with a correlator $\left\langle\eta(\boldsymbol{r}) \eta\left(\boldsymbol{r}^{\prime}\right)\right\rangle=$ $\langle\eta\rangle^{2}+\left\langle\eta^{2}\right\rangle \delta\left(x-x^{\prime}\right)$. Equation (9) is then integrated in the "weak" disorder case [11], i.e., when the disorder intensity is much smaller than the dimensionless surface tension, in a system size of $L=512$ and 25 disorder realizations with $\epsilon=1$, and the forced-flow boundary condition $\nabla \mu=-\bar{v} \hat{y}$ is imposed at the bottom of the system [10]. All the values for the average front velocity have been normalized to a reference value $v_{0}=0.002$, which corresponds to the highest value studied in this paper.

Numerical results.- Figure 2 shows the avalanche size and duration statistics calculated using a threshold $c_{\mathrm{th}}=3$. We observe that the probability distributions tend to a power-law as $\bar{v}$ is decreased, due to the divergent cutoff [cf. Eq. (4)]. We estimate the exponents $\tau_{s} \simeq 1.54(5)$ and $\tau_{T} \simeq 1.62(6)$ from the scaling of the data. Despite the smallest velocity we were able to reach $\bar{v}=v_{0} / 40=5 \times 10^{-5}$, which is still far from zero, the scaling region is reasonably good. According to our scaling theory both exponents should exactly coincide at the critical point, which is consistent with the numerical values within the error bars. We also plot a direct estimate of the divergent avalanche size cutoff in good agreement with Eq. (4). 



FIG. 4: (Color online) Scaling of the front roughness in the phasefield simulations for $L=512$ in the low velocity $\bar{v}=v_{0} / 40$ limit. (a) Structure factor $\mathcal{S}(k, t)$ at different times giving a spectral roughness exponent of $\alpha_{s}=1.45(6)$ [14]. (b) Data collapse of the local width according to a super-rough scaling for $\alpha=1.50(3)$, $\alpha_{\text {loc }}=1.0$, and $z=2.09(6)$.

We also find an excellent agreement with our prediction in Eq. (6) for the scaling relation between size and duration of an avalanche, $\langle s(T)\rangle \sim T^{\gamma}$. In Fig. 3]we plot both avalanche size and lateral extent $v s$ time for two typical velocities. For the lowest velocity we studied $\bar{v}=v_{0} / 40=5 \times 10^{-5}$ we estimate $\gamma \simeq 1.09(7)$, which is to be compared with $\gamma=1$ from Eq. (6) in $d=1$. This can also be compared with the scaling relations between the avalanche exponents $\tau_{s}$ and $\tau_{T}$ given by Eq. (5). Substituting the numerical values $\gamma=1.09$ and $\tau_{T}=1.54$ we predict $\tau_{t} \simeq 1.60$ in good agreement with the numerical result (cf. Fig. 22). Note that the scaling theory is expected to be exact only at the critical point $\bar{v}=0$, which is not actually reached with our phase-field model results. However, the singularity is strong enough to lead to effective exponents for velocities within a critical region $\bar{v} \leq L^{-2}$.

At variance with Rost et al. [4], who only monitored avalanches in the global velocity time series $\bar{v}(t)$, here we are actually looking at active sites that participate in an avalanche and, therefore, we are able to check the validity of the scaling law $\langle\ell\rangle \sim T^{\delta}$. The typical lateral extent is predicted to scale with avalanche duration with an exponent $\delta=1 /\left(\alpha_{\text {loc }}+\right.$ $d / 2)=2 / 3$ in the high velocity regime for $d=1$ [4], in excellent agreement with our numerical estimate in Fig. 3. However, for low velocities we predict $\delta=1 / z=1 / 2$ with the dynamic exponent $z=2$ in the static limit. A strong proof of a distinctive behavior as the front velocity is decreased can be readily seen in Fig. 3 Our numerical simulations indicate that $\gamma \rightarrow 1$ and $\langle\ell\rangle \sim T^{1 / 2}$ as $\bar{v} \rightarrow 0$, pointing out that the dynamics is controlled by the static critical point at $\bar{v}=0$.

On the other hand, we have also estimated the scaling of the interfacial fluctuations for states close to the static limit. This provides an independent check of the validity of our scaling relations in Eqs. (5) and (6) connecting avalanche and roughness exponents. From the structure factor $\mathcal{S}(k, t)=$ $\left\langle\left|h_{k}(t)\right|^{2}\right\rangle$ and local width in Fig. 4, we estimate the global, local, and spectral roughness exponents [14] $\alpha=1.50(3)$, $\alpha_{\mathrm{loc}}=1, \alpha_{\mathrm{s}}=1.45(6)$, respectively, and the dynamic exponent $z=2.09(6)$ in excellent agreement with our scaling theory for the pinned state.

Finally, we claim that the existence of a singular behavior as $\bar{v} \rightarrow 0$ and the extent of the critical region $\bar{v} \leq L^{-2}$

\begin{tabular}{c|cccc}
\hline \hline $\bar{v}$ & $\xi_{\times} / L$ & $\alpha$ & $z$ & $\gamma$ \\
\hline$v_{0}$ & 0.1 & 1.33 & 3 & 1.28 \\
$v_{0} / 5$ & 0.23 & 1.35 & 2.8 & 1.21 \\
$v_{0} / 20$ & 0.48 & 1.41 & 2.3 & 1.13 \\
$v_{0} / 40$ & 0.64 & 1.50 & 2.09 & 1.09 \\
\hline \hline
\end{tabular}

TABLE I: Correlated extend and effective scaling exponents from phase-field simulations at different velocities in as system of size $L=512$. The fronts are always super-rough with $\alpha_{\text {loc }}=1$ [14].

explain earlier numerical observations [5, 10] that reported a dependence of the critical exponents $\alpha(\bar{v})$ and $z(\bar{v})$ with the velocity in numerical results of forced-flow imbibition in finite systems. Table [ summarizes the different interfacial scaling exponents we observed for different velocities. We observe that as the static limit is approached the dynamic exponent $z \rightarrow 2$ and the roughness exponent $\alpha \rightarrow 3 / 2$, as corresponds to the pinned state.

To conclude, we have studied avalanche dynamics in forced-flow imbibition in the pinning limit $\bar{v} \rightarrow 0$. A scaling theory relating the roughness of the front with the avalanche dynamics has been developed in excellent agreement with numerical results. Our scaling analysis is based on the presence of long-range correlations due to the divergent characteristic length scale at $\bar{v} \rightarrow 0$. From an experimental point of view, it would be of great interest to explore the pinning limit. In the experimental setup of a Hele-Shaw cell [6], this limit may be achieved by putting the cell at an angle so that gravity plays a role. This setup should produce fronts near pinning. Note that already in the case of having a correlation length of about a $30 \%$ of the system size, the effect of the critical point should show up in a drift of the measured scaling exponents (see Table I). Alternatively, fluctuations around the critical point $\bar{v}=0$ could also be experimentally tested by setting the cell at an angle, so that the front is pinned, and then study how the system responds to a small angle variation. In this configuration we expect the front to jump from one pinned state to another following a relaxation dynamics described by Eq. (8) driven by avalanches described by Eqs. (5) and (6).

We thank R. Planet, J. Ortín and S. Santucci for discussion on their experimental work. This work is supported by the DGI (Ministerio de Educación y Ciencia, Spain) through Grant Nos. FIS2006-12253-C06-04 and -05.

[1] K. J. Maloy et al., Phys. Rev. Lett. 96, 045501 (2006); D. Bonamy, S. Santucci, and L. Ponson, ibid. 101, 045501 (2008).

[2] A. Daerr and S. Douady, Nature (London) 299, 241 (1999).

[3] D. S. Fisher, Phys. Rep. 301, 113 (1998).

[4] M. Rost et al., Phys. Rev. Lett. 98, 054502 (2007).

[5] M. Alava, M. Dubé, and M. Rost, Adv. Phys. 53, 83 (2004).

[6] R. Planet, S. Santucci, and J. Ortín, Phys. Rev. Lett. 102, 094502 (2009).

[7] E. Pauné and J. Casademunt, Phys. Rev. Lett. 90, 144504 (2003). 
[8] M. Dubé et al., Phys. Rev. Lett. 83, 1628 (1999).

[9] A. Hernández-Machado et al., Europhys. Lett. 55, 194 (2001).

[10] T. Laurila et al., Eur. Phys. J. B 46, 553 (2005).

[11] T. Laurila, M. Pradas, A. Hernández-Machado, and T. AlaNissila, Phys. Rev. E 78, 031603 (2008).

[12] M. Pradas and A. Hernández-Machado, Phys. Rev. E 74,
041608 (2006).

[13] J. Soriano et al., Phys. Rev. Lett. 95, 104501 (2005).

[14] J. J. Ramasco, J. M. López, and M. A. Rodríguez, Phys. Rev. Lett. 84, 2199 (2000). 